\title{
Special Issue in Clinical Radiology
}

Revised 18 September 2013

Accepted 20 September 2013

The Chinese Journal of Radiology was founded in 1953, and it is the first and the most influential comprehensive professional journal in medical imaging in China. The journal has played an important role in promoting research, education and clinical practice in the past 60 years, and it has been considered as the journal of choice by authors and readers in the field of clinical radiology and medical imaging. With the rapid development of medical imaging sciences and advancement in clinical services, the Chinese Journal of Radiology has experienced a fast growth in both quality and quantity in the recent years. Due to its being a journal published only in Chinese language, the accessibility of these high quality papers to non-Chinese speaking radiologists has also been limited. We therefore cooperated with the Journal of $X$-ray Science and Technology to launch a special issue in Clinical Radiology. We hope that the Clinical Radiology will provide Chinese radiologists a forum to share research findings and clinical experiences with overseas peers, and vice versa. It is my pleasure to serve as the guest editor of this special issue. 